\title{
Communication, action et technologie : quelle évaluation des pratiques?
}

Serge Agostinelli

\section{(2) OpenEdition}

1 Journals

Édition électronique

URL : http://journals.openedition.org/communicationorganisation/1368

DOI : 10.4000/communicationorganisation. 1368

ISSN : $1775-3546$

Éditeur

Presses universitaires de Bordeaux

Édition imprimée

Date de publication : 1 décembre 2010

Pagination : 17-28

ISBN : 978-2-86781-743-4

ISSN : $1168-5549$

\section{Référence électronique}

Serge Agostinelli, «Communication, action et technologie : quelle évaluation des pratiques ?», Communication et organisation [En ligne], 38|2010, mis en ligne le 01 décembre 2013, consulté le 01 mai 2019. URL : http://journals.openedition.org/communicationorganisation/1368 ; DOI : 10.4000/ communicationorganisation. 1368 


\title{
Communication, action et technologie : quelle évaluation des pratiques?
}

\author{
Serge AGOSTINELLI
}

Le but de cet article est de proposer une réflexion sur le rôle que peuvent jouer sur les organisations, les processus et procédures d'évaluation des pratiques professionnelles dans le contexte des technologies de l'intelligence.

$\mathrm{La}$ nécessité de reconnaître et d'évaluer les pratiques cruciales qui témoignent du rôle de plus en plus fort que constitue la maîtrise d'un véritable capital humain dans les organisations demande d'envisager plusieurs types d'évaluation: le premier autorise un contrôle et une appréciation globale d'une pratique à partir d'un référent stable et prédéfini; le deuxième permet une appréciation globale et partielle des changements de procédures et l'adaptation aux normes et contraintes pendant la reconnaissance des tâches à effectuer et les actions liées qui constituent la pratique observable; la troisième favorise la compréhension des ajustements des processus humains à l'intérieur même de la pratique qui font évoluer les référentiels métiers.

\section{La notion de pratique}

La notion de pratique définit aujourd'hui ce qui permet aux individus de «faire » dans un rapport au contingent. Elle a pour double fonction de produire les structures à travers les procédures explicites qui donnent corps à l'activité et de construire du sens à travers les processus tacites qui expliquent l'action. Dès lors, nous envisageons ici, la pratique professionnelle comme l'articulation entre des processus de l'ordre de l'humain qui organisent les connaissances subjectives et le savoir-être, et les procédures de l'ordre du métier qui dotent d'une structure, les connaissances objectives et le savoirfaire.

Cette articulation fait de la pratique professionnelle un ensemble dont il est impossible de dégager ou d'isoler les opérations cognitives de l'activité humaine, de plus, elle ne nous renseigne pas sur la nature individuelle ou

01. Serge Agostinelli est Professeur des Universités en Sciences de l'Information et de la Communication à I'Université Paul Cézanne - Aix-Marseille 3. II est chercheur au LSIS, Laboratoire des Sciences de l'Information et des Systèmes, UMR CNRS 6168. Mail : serge.agostinelli@univ-cezanne.fr 
collective d'une pratique. En d'autres termes, une pratique professionnelle peut-elle être individuelle ou est-elle obligatoirement collective? $\mathrm{Si}$ on explique une pratique à travers des critères qui impliquent les seuls individus avec leurs qualités, leurs objectifs, leurs croyances et leurs actions alors on renvoie effectivement à une pratique contingente.

La pratique professionnelle n'est alors pas autre chose que la somme des procédures qui la composent. Elle est une juxtaposition de pratiques qui sont nécessairement premières dans l'ordre professionnel avec trois implications pour leur évaluation:

- Les pratiques sont dépendantes des activités individuelles et non pas inversement

- L'évaluation des activités collectives n'est jamais ultime et doit être expliquée à son tour par des évaluations individuelles

- Les évaluations individuelles ne doivent jamais être formulées avec des termes relatifs à l'évaluation d'un collectif.

En revanche, si comme nous le proposons, la pratique professionnelle est définie comme étant une spécification formelle d'une conceptualisation partagée alors il existe des savoir-être qui précèdent le savoir-faire des individus dans l'ordre explicatif. Ceci renvoie à un autre type d'évaluation des liens et des attitudes avec la profession. Ces deux types d'évaluation ne sont pas symétriques car cette seconde renvoie aussi à trois principes mais opposés:

- Les pratiques individuelles sont liées à des pratiques sociales et collectives

- L'évaluation des activités individuelles n'est jamais ultime et doit être expliquée par les activités collectives

- L'évaluation des activités collectives ne peut jamais être formulée avec des termes relatifs aux activités des individus.

Loin de nous l'idée de dire qu'une évaluation est meilleure que l'autre. Cette introduction montre que l'évaluation n'est pas unique et quelle qu'elle soit, elle a ses intérêts, ses objets, ses limites.

\section{Les types d'évaluation}

La première approche pratique de l'évaluation est sommative. Dans la ligne directe du paradigme originel de la docimologie et de la mesure (Pieron, 1963), elle participe au contrôle de l'activité qui s'opère à partir d'un référent stable prédéfini. Elle constate ainsi les déviances ou les concordances à l'intérieur d'un espace homogène et renvoie naturellement aux méthodes expérimentales de mesure. Cette évaluation se définit comme une évaluation finale et externe qui réside dans une appréciation globale des changements survenus dans une pratique. C'est-à-dire qu'elle certifie que l'individu a bien les compétences requises dont la pratique professionnelle a besoin: par exemple, l'évaluation d'un personnel par rapport à sa fiche de poste.

Elle consiste en une mesure en trois étapes successives:

- L'observation permet de recueillir de l'information 
- L'interprétation ou jugement de l'information recueillie

- L'exploitation de l'information interprétée et décision de l'évaluateur.

L'objet de cette évaluation est la pratique accomplie, la procédure finie, exécutée. Elle s'intéresse donc essentiellement au résultat et aux rapports entre ceux-ci et les procédures. Ses fonctions visent à établir un bilan des activités visibles et valorisent particulièrement les comportements correspondant aux objectifs, aux normes, aux projets préalablement définis. L'intérêt majeur de cette évaluation réside dans l'explicitation d'un référentiel constitué a priori, unique et stable. Toutefois, ce type d'évaluation limite abusivement l'observation de l'activité au résultat de celle-ci. Globalement, ce type d'évaluation ne tient pas compte du déroulement de l'activité et si elle permet une information éventuelle des déviances a posteriori, elle ne permet pas d'informer sur les mécanismes cognitifs individuels ou collectifs mis en œuvre.

La deuxième évaluation est dite formative. Elle est introduite par Scriven (1967) avec pour objectif, les ajustements successifs lors du développement et l'expérimentation de nouveaux curricula de formation. Elle peut se définir comme une évaluation interne des procédures et réside dans une appréciation globale ou partielle de l'évolution des pratiques en cours dans une activité. Le bénéfice en revient à l'instance, au décideur, qui utilise la pratique et qui doit améliorer celle-ci pendant qu'elle fonctionne: par exemple, l'évaluation d'un service par une gouvernance d'institution ou d'un décideur qui conduit un changement. C'est une évaluation continue composée de plusieurs sousévaluations effectuées en fonction d'un critère objectif. Elle intervient, en principe, au terme de chaque tâche, qui a pour objet d'informer à la fois le personnel et les instances du degré de maîtrise atteint et, éventuellement, de découvrir où et en quoi un personnel éprouve des difficultés d'exécution, en vue de lui proposer ou de lui faire découvrir des stratégies qui lui permettent de changer de pratique.

En termes d'activité, elle se définit suivant trois étapes essentielles:

- Le recueil d'informations concernant les progrès et les difficultés rencontrés par les individus

- Linterprétation des informations de façon à diagnostiquer les facteurs à l'origine des difficultés

- Ladaptation des activités en fonction de l'interprétation.

Pour passer de cette définition à l'élaboration d'une stratégie d'évaluation formative, il faut se référer à un cadre conceptuel qui permette de préciser:

- Les aspects de l'activité qu'il faut observer et les procédures qu'il faut utiliser dans le recueil des informations

- Les principes qui vont guider l'interprétation des données et le diagnostic des problèmes de réalisation de l'activité

- Les démarches à suivre dans l'adaptation des activités. 
Elle se caractérise aussi par la pluralité des référentiels mis en jeu et par la nécessaire négociation de ces référentiels. Cette négociation constitue la trace même de l'évaluation formative car les pratiques professionnelles se développent dans le mouvement des interactions de référentiels qui induisent les ajustements nécessaires à l'adaptation des procédures en situation.

Ses modalités ont toujours une fonction d'ajustement, c'est-à-dire qu'elles assurent l'articulation entre les savoir-faire des personnes et ce qu'ils ont à faire. Son but est alors de fournir des informations qui permettent une adaptation de l'environnement professionnel aux différences individuelles dans l'activité. Le fonctionnement d'un individu, son comportement, constituent l'objet privilégié de l'évaluation formative et trois fonctions permettent cette adaptation:

- La valorisation des savoir-faire pertinents au référent renforce les processus cognitifs de l'individu qui ont permis la mise en œuvre de procédures correctes

- La modification des savoir-être en décalage avec les normes reconnues permet à l'individu de modifier les comportements qui lui semblent inadaptés

- L'ajustement externe permet une diminution de la déviance entre les objectifs et les stratégies utilisées pour les atteindre.

Le premier intérêt de l'évaluation formative est que les normes du savoirfaire ne sont pas figées, elles se construisent en étant multiréférenciées. Les critères de réalisation de l'activité et de réussite sont explicités. Le second intérêt, réside dans le fait qu'elle prend en charge une complexité qui ne se laisse pas réduire. Ce qui signifie qu'elle ne peut fonctionner que par l'interaction de plusieurs référents, celui de l'individu, celui des autres, mais également le référent qui se construit dans l'interaction.

Sa limite est d'envisager l'activité comme centrale, ce qui a pour effet de privilégier les procédures observables pour comprendre les pratiques. Les décisions qui en découlent sont d'ordre strictement opératoire et vise uniquement à réguler les conditions de l'activité. L'évaluation formative est donc un ensemble de procédures indiquant non seulement si les objectifs visés sont atteints, mais aussi de quelle façon ils le sont. Elle englobe donc, non seulement les résultats, mais aussi les méthodes, les décideurs, les personnels et tous les éléments qui interviennent dans le déroulement de la pratique. Elle intervient en cours d'activité avec l'intention de rétroagir sur la pratique.

La troisième évaluation associe le terme de régulation. Elle apparaît dans le cadre d'une démarche d'évaluation des processus qui activent les connaissances et savoir-être d'un individu, de ses stratégies d'action et de réalisation des tâches (Bonniol, 1988). Le terme de régulation a donc pour intention de centrer l'évaluation sur l'individu agissant sur les processus qui adaptent le savoir-faire et plus particulièrement sur les processus de régulation. C'est-à-dire qu'après l'organisation de conditions de l'activité, c'est sur le fonctionnement cognitif de l'individu, en terme de stratégies que 
l'on s'interroge, et surtout que l'on souhaite que s'interroge et opère l'individu lui-même.

La régulation peut être définie suivant deux approches: d'une part, en cybernétique, elle est une notion de mesure; d'autre part, en physiologie, elle est une notion d'équilibre. Toutefois, ces deux positions ne sont pas antinomiques, des passerelles existent. En fait, les différences résident dans la façon dont les interactions sont mises en œuvre entre le système cognitif d'un individu (savoir-être, connaissances, processus) et le système global de l'activité envisagé comme un complexe d'actions intelligibles (Le Moigne, 1990). En cybernétique, le système de régulation permet de recueillir les informations sur les entrées et/ou les sorties; de déterminer en fonction de l'écart probable des sorties par rapport à une norme, les variables de commandes qui lui permettent de se rapprocher de cette norme. La régulation est limitée ici, à sa fonction informationnelle. En revanche, avec l'approche physiologique, la régulation assure la constance des caractères du milieu intérieur d'un individu en dépit des variations du milieu extérieur. C'est l'homéostasie qui singularise le vivant, c'est-à-dire, le jeu des régulations qui fait apparaître la notion d'état stationnaire ou d'équilibre dynamique (Morin, 1977).

L'évaluation régulation trouve donc sa source dans une approche systémique qui met en avant une interaction d'éléments constituant une entité ou une unité globale. Les interrelations entre éléments, événements, ou individus (individu au sens de sous-système), sont organisationnelles (Morin, 1977). Dans cette définition apparaît l'idée d'adaptation par interaction dynamique et l'idée de boucle rétroactive est fondamentale. Dès lors, l'évaluation régulation peut se définir comme un processus d'information à l'intérieur même de la pratique grâce à une rétroaction permanente. L'évaluation agit sur la pratique et lui permet de réguler, de mieux s'adapter... laquelle pratique agit à son tour sur l'évaluation. L'interaction entre la pratique et l'évaluation permet alors une régulation réciproque capable de faire évoluer les référentiels. Les régulations intéressent en particulier les opérations mentales qui construisent les stratégies d'adaptation que l'individu doit mettre en ouvre dans l'activité.

Sa fonction essentielle est de mettre en rapport interactif les deux systèmes dynamiques: la pratique professionnelle globale et individuelle avec une évaluation globale et individuelle, pour favoriser une régulation réciproque à la fois interne et externe. Le premier intérêt de l'évaluation régulation est qu'elle renvoie à l'individu ce qu'il fait pendant qu'il le fait. On est ici au stade de la régulation au cours de l'activité, ce qui lui permet d'ante-agir en fonction de l'évaluation qu'il effectue sur les résultats attendus de ses actions cognitives. Le second intérêt réside dans son rôle organisationnel de l'action cognitive qui modifie le statut de la pratique ancienne en élément structurant d'un nouveau savoir-faire. Les limites sont dans les ambitions annoncées, vouloir cerner les stratégies cognitives suppose que l'on dispose d'un modèle 
général de l'action mentale, d'une grille de lecture de ces opérations telles qu'elles sont susceptibles d'être.

En conclusion, pour évaluer les pratiques professionnelles, nous avons le choix non réductible entre les trois options en fonction de ce qui nous intéresse dans la pratique: les processus cognitifs, les procédures métier, l'activité individuelle ou prise dans une globalité. Aujourd'hui, la difficulté réside dans l'approche holiste des pratiques avec laquelle, l'évaluation ne peut pas se limiter à un type d'objets observé. La pratique professionnelle est insérée dans un contexte social et historique, elle est partagée par les membres d'une culture. Dès lors, les processus cognitifs ne peuvent être dissociés de la dimension intersubjective du savoir-faire et les questions de connaissances communes, du partage d'opinions, de l'information pour autrui et avec autrui se caractérise par la perception du contexte, des stratégies cognitives et de l'activité humaine. Ce rapprochement qui fonde particulièrement l'évaluation régulation relève de trois caractéristiques:

- Il est significatif et ne peut être compris sans recours à des éléments liés au contexte pragmatique

- La signification change avec le contexte car l'évaluation se réfère à des objets différents

- Il comporte des indicateurs réflexifs sans valeur descriptive, les déictiques et les index qui marquent la dépendance contextuelle.

La pratique et l'évaluation relèvent donc d'un système cognitif indexical dans un contexte socio-informationnel organisé.

\section{Pratique d'action intelligente}

$\mathrm{Au}$ sein de ce système cognitif indexical, la pratique professionnelle devient l'aboutissement d'une conception d'un monde local, d'une prise d'information et d'une modélisation de celle-ci pour l'action. La pratique d'action intelligente décrit alors l'élaboration d'un savoir-faire par toute forme de raisonnement descriptible a posteriori, d'une stratégie d'action proposant une correspondance adaptée entre une situation perçue et un projet conçu par le métier auquel on s'intéresse.

L'indexicalité (Garfinkel, 1967) positionne donc la pratique professionnelle dans un groupe particulier de professionnels comme un processus d'accomplissement. C'est le geste professionnel en train de se construire qui permet la construction des connaissances situées qui, de manière réflexive entretiennent, vérifient, modifient les procédures de l'activité. La pratique est envisagée ici comme un processus interactionniste et praxéologique, constitutivement lié aux situations qui ordonnent la production, la diffusion et l'appropriation des informations, des connaissances professionnelles au sein d'un espace collectif qui met en relation tous les acteurs de sa formalisation.

Dès lors, la pratique professionnelle dont les activités sont des indicateurs, pose la question épistémologique de savoir comment considérer: 
- Une action humaine dans son aspect objectif et accompli comme une connaissance

- Une évaluation comme moyen d'information d'une part, outil de connaissance d'autre part.

L'évaluation s'efforce ici de caractériser toutes les possibilités et les contraintes contenues dans l'activité professionnelle, mais aussi les effets produits par sa mise en œuvre. Ce n'est que progressivement que l'évaluation laissera transparaître sa dimension subjective qui, dans un premier temps, surprend essentiellement par son purisme, voire son abstraction.

En effet, voulant révéler la spécificité d'une pratique, le risque est de rechercher l'action intelligente et immédiate, quasi automatique, où la nécessité de sa formalisation se confond avec l'élaboration de cette formalisation. En revanche, distinguer les aspects de la construction d'une pratique peut donner une heuristique pour d'autres formes de formalisation ou d'évaluation: le contexte dans lequel apparaît celle-ci, les usages qui agissent sur cette apparition et enfin, les actions liées.

\section{Une fonction organisatrice de l'évaluation}

L'évaluation a une fonction organisatrice fondamentale, tant du point de vue de l'attribution de sens par l'individu à une situation de travail que de la racontabilité de l'action. Ce sont les gestes professionnels et leurs mises en relation avec les situations, c'est-à-dire leur accoutability qui génère les connaissances situées. La pratique est donc une médiation entre un individu, son activité, l'autre et son monde, c'est-à-dire, une représentation d'un univers local, principalement centré autour d'un groupe limité de personnes (entreprise, atelier, service, etc.). Cette médiation est, pour une large part, implicite car elle fait intervenir des allant de soi; elle est socialisée car elle est interactivement partagée entre les membres du groupe qui construisent des significations communes et intersubjectives; c'est-à-dire un système commun de communication centré sur une cognition de groupe, sur la construction de savoir-faire nouveaux mais collectivement élaborés et collectivement médiatisés.

\section{Une fonction informationnelle}

Selon nous, la pratique professionnelle dépend de deux choses:

- L'organisation de la base de connaissances dont dispose chaque individu, (on fait l'hypothèse que l'individu dispose de savoir et de savoir-faire, il a des pratiques personnelles, même si elles ne sont pas des pratiques reconnues)

- La relation entre l'organisation de la base de connaissance et les savoirfaire qui entrent dans une pratique particulière.

On ne parle plus alors d'une pratique à évaluer suivant un système autoorganisant mais suivant des transformations caractéristiques des composantes cognitives, affectives, imaginatives du métier. Il ne s'agit plus, de chercher 
des explications mais les règles d'un système commun autorisant la compréhension ou la création du sens à l'action humaine. Ici, les savoir-faire vont se spécifier dans un domaine particulier et l'évaluation d'une pratique devient le mode de communication des connaissances fonctionnelles. On n'évalue plus une expertise mais des critères opérationnels pour le choix des informations indexées à une situation de terrain. C'est d'ailleurs l'analyse de la situation de travail qui va autoriser les inférences sur la pratique et les inférences sur l'organisation et l'utilisation des connaissances effectivement mises en jeu dans cette situation. Par rapport à un ensemble de situations dans un domaine d'activité, on peut donc observer un certain nombre de pratiques qui visent à ce que cette collection d'informations mette à jour des invariants fonctionnels généralisables pour ouvrir le champ des connaissances sur une pratique particulière. C'est la gestion individuelle de ces invariants qui permettra à chacun de prendre des décisions de l'ordre de la stratégie sans pour cela avoir une vision d'immédiateté.

On passe alors, d'une évaluation comme moyen de production d'informations à un outil de gestion de la structure d'une activité qui donne une heuristique de contrôle des savoirs déjà là dont l'étude permettrait d'éclairer l'évolution et les changements de pratiques utilisées par les individus pendant l'activité. Les savoirs déjà là réfèrent d'une façon générale à la régulation de l'action: planification ou anticipation de l'action; auto-guidage de l'exécution de l'action; évaluation des résultats de l'action. Cette gestion de l'action peut être considérée comme un ensemble de stratégies d'attribution de sens, de significations visant à établir des liens entre les informations dont dispose l'individu et les inférences qu'il réalise. Les informations sur le contrôle de l'action devraient donc être centrales dans l'approche concernant le recueil d'informations sur une pratique professionnelle, dès lors qu'il favorise l'accès aux savoir-être impliqués dans la mise en œuvre des pratiques.

\section{Une fonction de régulation}

L'évaluation d'une pratique professionnelle devrait, pour nous, répondre à trois attentes des acteurs:

- Une attente en termes de valorisation car la pratique ne se construit pas sans une reconnaissance de certains savoir-faire, des normes et des règles qui sont utilisés pour indiquer les bons gestes professionnels, ou du moins, la progression vers ceux-ci. La pratique est d'abord un processus de satisfaction.

- Une attente en termes d'information qui va permettre le repérage, la reconnaissance des procédures, des routines à pérenniser. La construction d'une information pertinente à une pratique implique une reconnaissance des modèles praxéologiques et théoriques qui la sous-tendent, pour permettre leur objectivation et une valorisation pour permettre cette reconnaissance.

- Une attente en termes de réajustement car elle est intermédiaire entre l'intérêt porté aux savoir-être et l'intérêt porté aux savoir-faire. 
Ces trois attentes qui semblent révélatrices de la dynamique des fonctions organisatrices et informationnelles vont construire chez l'individu, les règles et leurs critères observables qui lui permettront d'évaluer le savoir-faire juste à travers des procédures correctes d'atteinte du but en fonction des informations avec lesquelles il doit réaliser son activité.

\section{Pratique d'action et technologies}

En regard des types d'évaluation évoqués plus haut, les technologies de l'intelligence, c'est-à-dire les technologies d'information et communication qui amplifient les processus relationnels ou cognitifs, devraient participer de l'évaluation régulation sans toutefois évacuer les deux autres. En fait, il s'agit pour nous de savoir quels sont les objets d'une évaluation des pratiques qui se construisent à travers les relations interindividuelles et collectives. Celles-ci constituent la genèse des situations technologiques qui portent l'action.

Le développement actuel de technologies nous conduit aujourd'hui, à la réduction de l'action collective et de l'organisation humaine à un ensemble d'actions individuelles. Le collectif est alors amalgamé à de l'interindividuel, dans la mesure où les actions collectives sont réduites à un assemblage de négociations, de conversations ou encore à une combinaison de prêt à agir, c'est-à-dire à des ressources individuelles partagées et utiles à la décision, à la coordination, à l'action. Il n'est que d'observer le développement des réseaux sociaux numériques, des blogs et autres outils de présence à distance. Dès lors, comment évaluer d'une façon externe (type 1) des pratiques le plus souvent tacites, relevant de procédures relationnelles implicites dont la communication est porteuse et constitue la base de discussion des actions possibles. Une analyse externe conduirait inévitablement à envisager la technologie comme une finalité du contrôle de l'action. Les objets de l'évaluation sont alors, les procédures qui déterminent les actions du quotidien et génèrent des décisions pour des actions futures. Lintérêt est une logique utilitariste propre à un domaine particulier: la politique, l'économie, les sciences... En retour, ces évaluations structurent la Société de l'Information par des "prêts à agir " utilisables dans les situations quotidiennes. Les décisions sont toutefois instanciées par les points de vue disponibles à un moment de l'histoire de notre société. Les activités humaines sont inscrites dans l'infrastructure matérielle qui détermine la compréhension des décisions et des relations. Le modèle de communication est à la fois segmenté et cumulatif, on est dans de l'événementiel.

En revanche, envisager les pratiques avec les technologies de l'intelligence comme un système socio-technico-informationnel indexé de mise en relation des outils, des pratiques, d'une situation et d'un contexte permet de dépasser une évaluation des technologies comme simple évaluation de moyens. L'évaluation est envisagée sous la forme d'une collecte d'informations reliées qui constitue un système périphérique de la maîtrise de notre société. Cette 
évaluation est alors autonome par rapport au quotidien et aux domaines d'application qui assurent les actions de prise de décision, d'intentionnalité ou d'organisation. Elle organise les pratiques en système anticipatif où les actions sont le résultat d'une Société de la Communication fondée sur la reconnaissance des relations et des situations d'usage. C'est un modèle de communication à la fois fonctionnel et contextuel. Utiliser les technologies de l'intelligence c'est construire de la relation. L'évaluation relève ainsi d'une étude globale des situations qui président à la manifestation des usages et interactions souhaitées... L'évaluation oriente l'intentionnalité, la réflexion et change l'activité humaine. Elle déclenche in fine, une activité intellectuelle fondée sur l'intelligibilité mutuelle, les actions situées et le partage des ressources. Les technologies sont alors inscrites dans l'organisation humaine pour produire et reconnaître leur monde, pour le rendre familier, acceptable à une société de la communication, de la relation.

\section{Conclusion}

Évaluer des pratiques - qu'elles soient individuelles ou collectives dans leurs dimensions spécifiques de construction d'un monde local connu et reconnu qui autorise les relations et le partage des ressources technologiques - nécessite de prendre en compte leur complexité non réductible à des usages, à des implantations ou à des modes de contrôle. C'est cette complexité qui définit les relations entre Communication, Action et Technologies. La fonction relationnelle de la communication qui relie les technologies à leurs pratiques devient l'indicateur du caractère collectif de l'action, ce qui donne du sens aux relations interindividuelles ou homme-outils-homme dans une situation collective préexistante.

\section{BIBLIOGRAPHIE}

BONNIOL Jean-Jacques, "Entre les deux logiques de l'évaluation, rupture ou continuité? » Bulletin de l'ADMEE, vol. 88, n 3, 1986, pp. 1-6.

GARFINKEL Harold, Studies in ethnomethodology. Cambridge: Polity Press, 1967, $304 \mathrm{p}$.

Le MOIGNE Jean-Louis, Modélisation systémique de la complexité. Paris : Dunod, 1990, $178 \mathrm{p}$.

MORIN Edgar, La Méthode 1 - La nature de la nature. Paris : Seuil, 1977, 399 p.

PIERON Henri, Examens et docimologie, Paris : PUF, 1963, 180 p.

SCRIVEN Michael, «The Methodology of evaluation ", in Perspectives of curriculum evaluation, TYLER Ralph, GAGNE Robert, SCRIVEN Michael, Chicago: Rand Aller Nally, 1967, pp. 39-83. 
Résumé : La pratique professionnelle est envisagée ici comme une solution construite, liée à un contexte d'action et d'usages. Son évaluation peut s'envisager selon trois options : un contrôle et une appréciation globale à partir d'un référent stable et prédéfini ; une appréciation globale et partielle des changements de procédures et l'adaptation aux normes et contraintes pendant la reconnaissance des tâches à effectuer et les actions liées qui constituent la pratique observable ; la compréhension des processus humains à l'intérieur même de la pratique qui fait évoluer les référentiels. Cette troisième option s'adapte particulièrement à la prise en compte des facteurs humains liés aux technologies de l'intelligence. Les pratiques sont ici, révélatrices d'un système socio-technicoinformationnel indexé.

Mots-clés : facteur humain, complexité, usage collectif

\begin{abstract}
The professional practice is considered here like a solution built and biding on a context of action and uses. Its evaluation can be considered according to three options: a control and an overall assessment starting from a stable and preset referent; an overall assessment and partial of the changes of procedures and the adaptation to the standards and constraints during the recognition of the tasks to be carried out and the actions biding which constitute the observable practice; the comprehension of the human processes inside even of the practice which makes evolve the reference frames. This third option adapts particularly to the taking into account of the personal elements related to technologies of the intelligence. The practices are, revealing here of an indexed social, technical and informational system.
\end{abstract}

Key-Words : Human factor, complexity, collective use 